\title{
Analysis of germanium epiready wafers for III-V heteroepitaxy
}

\author{
Ignacio Rey-Stolle , Enrique Barrigon, Beatriz Galiana, Carlos Algora \\ Instituto de Energia Solar, Universidad Politecnica de Madrid, ETSI de Telecomunicacion, Avda. Complutense s/n, 28040 Madrid, Spain
}

Keywords:

Al. Substrates

Al. Surface processes

A3. Metalorganic vapor-phase epitaxy

B2. Semiconducting germanium

\begin{abstract}
A B S T R A C T
Frequently, when growing III-V semiconductors on germanium substrates, unexpected differences between nominally identical substrates are encountered. Using atomic force microscopy (AFM), we have analysed a set of germanium substrates sharing the same specifications. The substrates come from the same vendor but different results come about in terms of the morphology of the epilayers produced by the same epitaxial routine (i.e. substrate $W$ produced epilayers with good morphology while substrate $W X$ produced epilayers with defects). The morphological analysis has been carried out on (a) epiready substrates; (b) samples after a high-temperature bake at $700{ }^{\circ} \mathrm{C}$; and (c) on the samples after a hydride $\left(\mathrm{PH}_{3}\right)$ annealing at $640{ }^{\circ} \mathrm{C}$. In the two first stages all substrates (both $W$ and $W X$ ) show the same good morphology with RMS roughness below $3 \mathrm{~A}$ in all cases. It is in the third stage (annealing in $\mathrm{PH}_{3}$ ) that the morphology degrades and the differences between the samples become apparent. After phosphine exposure at $640{ }^{\circ} \mathrm{C}$, the RMS roughness of both substrates approximately doubles, and their surface appears as full of peaks and valleys on the nanometre scale. Despite the general appearance of the samples being similar, a careful analysis of their surface reveals that the substrates that produce bad morphologies (WX) show higher peaks, and some of their roughness parameters, namely, surface kurtosis and the surface skewness, are considerably degraded.
\end{abstract}

\section{Introduction}

Germanium is becoming a substrate of increasing relevance in the field of III-V epitaxy. Today germanium wafers are mostly used in the manufacture of solar cells (both for space and terrestrial concentrator systems) though there are other emerging applications which are starting to gather momentum such as photodetectors, LEDs, magnetoresistive sensors and even HEMTs

- The main reason for this success is that germanium only shows a small lattice mismatch to gallium arsenide and thus meets the key criterion for high-quality III-V growth of GaAs and "GaAs-compatible" III-Vs, while it offers clear advantages over conventional GaAs substrates in certain applications. These extra benefits include high crystallographic perfection (dislocation free wafers), high mechanical strength (thinner wafers), slightly higher thermal conductance and, finally, germanium is an environmentally friendly substrate (i.e. it is As-free), easy to recycle Additionally, in the past Ge wafers had a substantially lower cost (roughly half that of GaAs wafers of the same size in 2007) but the price advantage currently seems to be vanishing.

Resulting from this extensive range of current and potential applications, in 2007 the number of $\mathrm{Ge}$ wafers produced accounted for more than $25.106 \mathrm{in}^{2}$ ( 2 million 4-inch wafers)
. Currently, the typical standard for this industry is a 4-inch wafer (for solar cell applications, which roughly represents around $95 \%$ of the market) but the development of 200 and $300 \mathrm{~mm}$ wafers is underway, mostly for nanoelectronic applications Mimicking the trends in GaAs-wafers, Ge vendors offer epiready substrates, that is, substrates with surfaces prepared to be directly used in an epitaxial reactor without any further cleaning. The details of the epiready process are vague but essentially it consists in a set of cleaning steps aimed at eliminating the metal contamination (coming from the cutting and polishing steps) and to form carbon and oxide layers which are readily removable by in-situ cleaning in a MOVPE reactor.

However, despite the market being mature and the substrate quality nominally high, many research groups still detect (sometimes) differences in growth results when switching from one batch of wafers to another, with nominally identical specifications. For instance, we have observed noticeable differences in the final morphology of the epilayers when applying the same growth sequence to nominally identical substrates (same manufacturer and characteristics) coming from different manufactured batches.

Germanium wafer manufacturers have recently began to pay some attention to this problem and several new techniques and analyses (TXRF, TD-GCMS, etc.) are being applied to the substrates in the quest for the elusive cause of these differences [2,4,5]. However, despite the fact that variability in growth is seen on germanium wafers, it is a problem that appears repeatedly in conversations between experts in the field, it has received 
practically no specific attention in the literature. Therefore, the main goal of this study is to contribute to the systematic analysis of these differences by following the evolution of the surface morphology across the stages of the heteroepitaxial process when applied simultaneously on "good" and "bad" substrates. Accordingly, in this study we have analysed (1) the initial epiready stage; (2) after the temperature ramping and heating process; and (3) after the pre-nucleation hydride exposure. The surface morphology in each of these steps for a set of different Ge wafers is monitored using atomic force microscopy (AFM) to detect when the divergence in morphological characteristics occurs and to identify the origin of the lack of reproducibility.

\section{Experimental procedure}

All samples were processed in a horizontal, low-pressure, AIX200/4 MOVPE reactor. The carrier gas was Pd-purified hydrogen with a total flow during the process of 14 standard litres per minute and a total pressure in the reactor of $100 \mathrm{mbar}$. Pure phosphine $\left(\mathrm{PH}_{3}\right)$ was used for the hydride annealing.

In this study we have used two different types of substrates (hereinafter $W$ and $W X$ ), whose key difference is the manufactured batch. Essentially, all substrates share the same main characteristics, a 4-inch wafer, oriented $(100)$ with a miscut of $6^{\circ}$ towards the nearest (111) plane, doped with gallium (p-type) up to a resistivity of $25+15 \mathrm{rr} \& l \mathrm{~cm}$, with no etch pits on their surface $\left(\mathrm{EPD}=\right.$ OcirT $\left.^{2}\right)$. Additionally, all wafers come from the same vendor and therefore must be nominally identical, apart from the batch-to-batch tolerances given by the manufacturer. The only noticeable difference is that they were cut from different ingots (i.e. they correspond to different manufacturing batches) and that our process for $\mathrm{II}-\mathrm{V}$ heteroepitaxy on $\mathrm{Ge}$ produces good morphology when applied to $W$ wafers and not-so-good morphology when applied to $W X$ wafers. The so-called good morphology corresponds to a featureless specular GalnP epilayer on the Ge substrate, which acts as a great template for further III-V-based device growth (solar cells in our case). The so-called not-so-good morphology occurs when the GalnP epilayer appears dotted with a certain amount of crystallographic defects randomly decorating the sample surface, which locally, in the areas free of defects, preserves the low roughness characteristic of the good morphology (some of these defects are discussed elsewhere in this volume [6]).

Full wafers are unpacked from their individual boxes in a laminar flow cabin, which provides locally class 10 conditions, and are cut into small pieces (several square centimetres) by cleaving along $\langle 110\rangle$ directions. After that, the samples are blown under ultra pure $(6 \mathrm{~N})$ nitrogen and transferred to a clean chamber filled with $6 \mathrm{~N} \mathrm{~N}_{2}$ next to the reactor. No other ex-situ cleaning was applied on the pieces.

For every experiment two samples (i.e. a piece of each type of wafer) are loaded into the reactor, so that $W$ and $W X$ substrates undergo the same process simultaneously. This approach guarantees minimum dispersion in the process for both samples but has the disadvantage of hindering the use of reflectance anisotropy spectroscopy (RAS), an excellent tool for in-situ surface analysis in an MOVPE environment. As we introduce two different samples in the reactor we cannot discriminate which sample is contributing to the RAS signal at each instant (i.e. we get a mixed RAS spectrum coming from the surfaces of two different wafers). Basically, the complete experiment sequence is similar to the first steps of the heteronucleation procedure

, which is essentially based on the growth of a thin GalnP layer as the III-V nucleation layers on germanium. The procedure consists of three steps: (1) it starts with a short bake at $350{ }^{\circ} \mathrm{C}$ in hydrogen for
1Omin; (2) the temperature is then raised and the samples baked at $700{ }^{\circ} \mathrm{C}$ again in hydrogen for $10 \mathrm{~min}$; and (3) samples are cooled down to $640{ }^{\circ} \mathrm{C}$ and are annealed in $\mathrm{PH}_{3}$ for $5 \mathrm{~min}$. At this point, in a normal heteronucleation process, the growth of GalnP would start by opening the valves of TMGa and TMIn and moving the phosphine flow to the desired value for the nucleation layer. However, in this study we have focused on phenomena which occur prior to nucleation so, just after the $\mathrm{PH}_{3}$ annealing, the heater is switched off and the sample is cooled to room temperature. The reactor is maintained under a light flow of $\mathrm{PH}_{3}$ until the temperature goes below $500{ }^{\circ} \mathrm{C}$, which usually takes around $3 \mathrm{~min}$.

In this work, three experiments have been developed by analysing the samples at three different points of the afore-described growth sequence, namely: (a) after cutting the pieces (epiready wafers); (b) after the high-temperature bake; and (c) after the hydride annealing.

The characterization of the samples was carried out using AFM with a Nanoscope Multimode Ilia (Digital Instruments, Veeco) instrument which was used in the tapping mode with standard silicon tips. A scan area of $2.5 \times 2.5 \mathrm{am}^{2}$ was used for the AFM analysis. Several different spots were scanned on each sample to better reflect the surface roughness and discard local irregularities. All images were subjected to a similar correction procedure, in order to remove undesired plane artefacts (tilt and bow) and random noise. This procedure consisted of an overall flattening correction through a two-order polynomial fit. The image processing and analysis was carried out using WSxM, a freeware scanning probe microscopy software based on MS-Windows

\section{Results}

Fig. 1 presents representative AFM images of the $W$ and $W X$ substrates for the three experiments carried out. Both height (in 3D) and amplitude images have been included for each sample and each experiment; height images provide the basis for quantitative analyses while amplitude images help identifying the morphological patterns mostly qualitatively. Table 1 summarizes the most relevant roughness-related parameters of the topography (height) AFM images in Fig. 1.

\section{Discussion}

\subsection{Morphology of epiready wafers}

Fig. la and g represent the topography AFM scans of the $W$ and $W X$ wafers at their epiready stage. Their corresponding amplitude scans are shown in Fig. $1 b$ and h. Ideally, this surface should reflect the step and terrace structure of a (100) plane miscut $6^{\circ}$ off towards the nearest (111) plane, composed of $(100)$ terraces around 27A wide separated by single ML steps [9], covered by a conformal oxide resulting from the epiready treatment and the exposure of the pieces to the atmosphere during the cutting process. The scale of the image $(2.5 \mathrm{am} \times 2.5$ (im) is not suitable to resolve the geometric features of this vicinal surface (however, smaller scans could not resolve the terrace-step structure either) which is, on the other hand, expected for a substrate covered by a conformal oxide. Power spectral density analysis (a typical analytical technique for extremely flat surfaces ) carried out on images la and $g$ showed the typical tapered curve characteristic of flat, isotropic surfaces. However, no spike was detected at the frequency corresponding to terrace spacings (i.e. again the geometrical features expected for this vicinal surface could not be determined). Nevertheless, the 

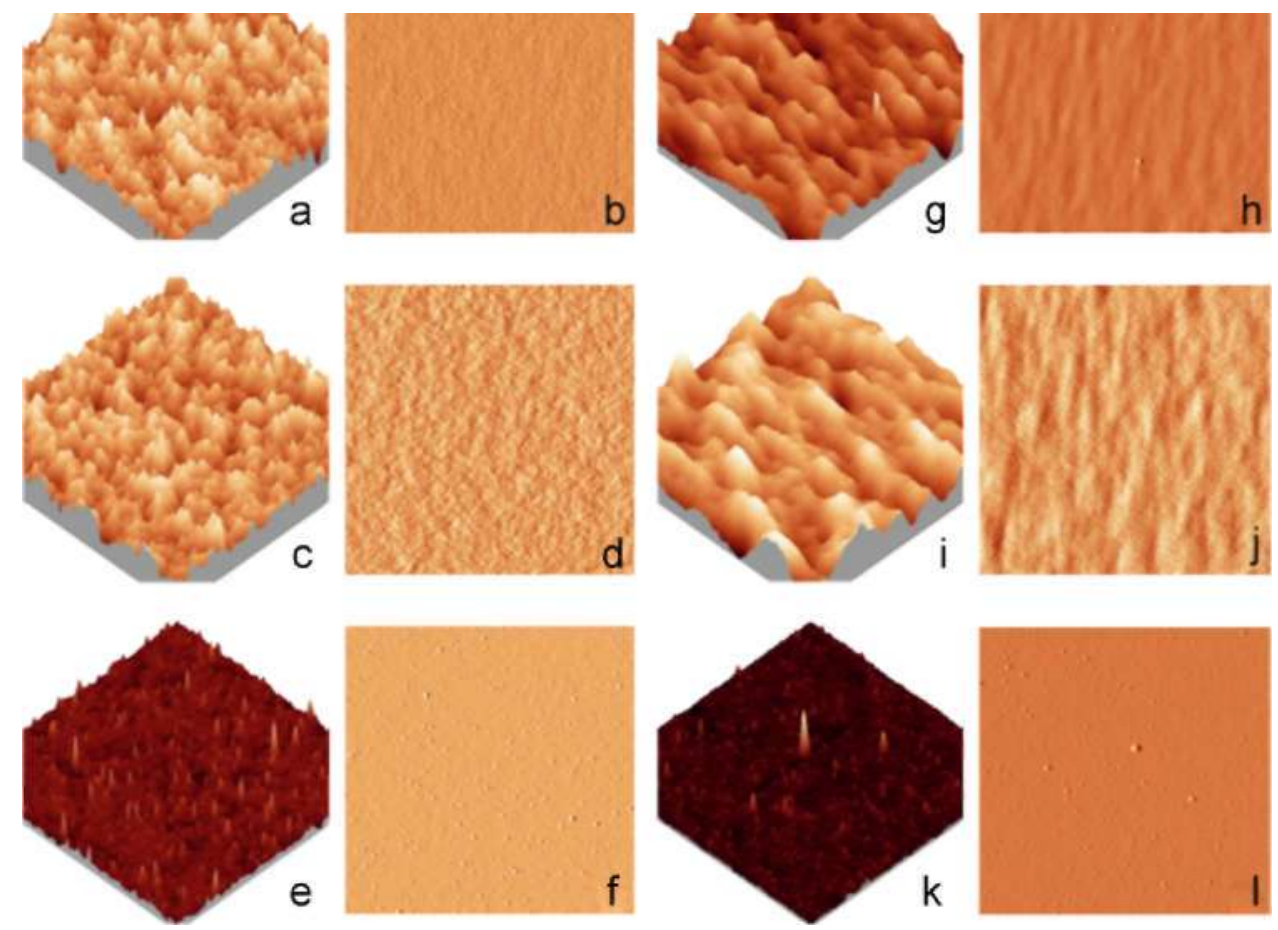

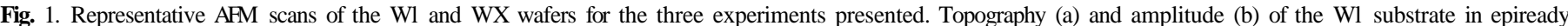

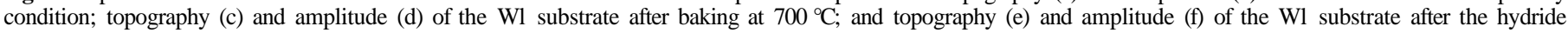
annealing at $640^{\circ} \mathrm{C}$. Images (g) to (1) are the counterparts for sample WX of figures (a) to (f).

Table 1

Main roughness-related parameters of the topography AFM images in Fig. 1

\begin{tabular}{llcc}
\hline Experiment & Surface parameter & Wl & WX \\
\hline Epiready samples & $R_{q}(\mathrm{~nm})$ & 0.26 & 0.13 \\
& $\mathrm{i} ?_{\mathrm{a}}(\mathrm{nm})$ & 0.21 & 0.10 \\
& $\mathrm{H}_{\mathrm{m}}(\mathrm{nm})$ & 1.15 & 0.46 \\
& $\mathrm{i}$ ?max $(\mathrm{nm})$ & 2.23 & 1.22 \\
& $R s k$ & 0.13 & -0.11 \\
& $R k u$ & 3.07 & 2.92 \\
Samples after baking at $700{ }^{\circ} \mathrm{C}$ & $R_{q}(\mathrm{~nm})$ & & \\
& $\mathrm{i} ?_{\mathrm{a}}(\mathrm{nm})$ & 0.26 & 0.13 \\
& $\mathrm{H}_{\mathrm{m}}(\mathrm{nm})$ & 0.21 & 0.11 \\
& $\mathrm{i} ? \mathrm{max}(\mathrm{nm})$ & 1.03 & 0.55 \\
& $R s k$ & 2.05 & 1.03 \\
& $R k u$ & -0.086 & -0.006 \\
& & 2.97 & 3.17 \\
Samples after annealing in $\mathrm{PH}_{3}$ & $R_{q}(\mathrm{~nm})$ & & \\
& $\mathrm{i} ?_{\mathrm{a}}(\mathrm{nm})$ & 0.61 & 0.74 \\
& $\mathrm{H}_{\mathrm{m}}(\mathrm{nm})$ & 0.38 & 0.42 \\
& $\mathrm{i} ? \mathrm{max}(\mathrm{nm})$ & 1.68 & 1.69 \\
& $R s k$ & 12.0 & 24.2 \\
& $R k u$ & 3.75 & 7.05 \\
& & 33.27 & 126.30 \\
\hline
\end{tabular}

Acronyms are as follows: $R_{q}$ is root-mean-square (RMS) roughness; J? is average roughness; $\mathrm{H}_{\mathrm{m}}$ is mean height; $\mathrm{J} ?_{\max }$ is maximum peak-to-valley height; $\mathrm{J}_{\mathrm{sk}_{\mathrm{s}}}$ is surface skewness; and $\mathrm{J} ?_{\mathrm{ku}}$ is surface kurtosis.

differences in morphology are evident between both samples. Wl shows a random pattern in height distribution (Fig. la) translating it into a featureless amplitude image (Fig. lb), similar to other AFM scans on epiready Ge wafers reported in the literature . On the other hand, $W X$ shows a softer height distribution forming a wavy pattern (Fig. 1g) which looks more apparent in the amplitude image (Fig. lh). It should be noted that the RMS roughness of Wl is $0.26 \mathrm{~nm}(\sim 1 \mathrm{ML})$ while that of $W X$ is $0.13 \mathrm{~nm}$ ( $\sim 0.5 \mathrm{ML})$. Thereby, both surfaces are extremely flat, with average roughness values $\left(\mathrm{R}_{\mathrm{a}}\right)$ below the threshold for a typical $\mathrm{Ge}$ epiready substrate $\left(R_{a}=5 A\right) \quad$; and with RMS roughness values also below those obtained by applying optimized chemical+ vacuum cleaning techniques on the substrates $\left(R_{q}=3-6 \mathrm{~A}\right)$

Therefore, we believe that these minor differences in morphology do not really provide significant evidence that could eventually justify differences in the morphology of epilayers grown on both surfaces.

\subsection{Morphology of wafers after baking at $700^{\circ} \mathrm{C}$}

Fig. lc and i present AFM scans of the $W$ and $W X$ wafers after the first initial low-temperature bake at $350{ }^{\circ} \mathrm{C}$ and the second high-temperature bake at $700{ }^{\circ} \mathrm{C}$. The corresponding amplitude scans are shown in Fig. Id and $\mathrm{j}$. The purpose of the hightemperature bake is twofold: (1) it is intended to desorb the native oxide and possible carbon contamination covering the samples and (2) it is meant to transform the single-step configuration on the surface of the epiready substrates, into the desired double-step surface structure needed for APB-free heteroepitaxial growth When the samples are cooled and extracted from the reactor a fresh oxide is again created, producing a situation very similar to that of epiready wafers. Therefore, the general features in Fig. $1 \mathrm{c}, \mathrm{d}$, $\mathrm{i}$ and $\mathrm{j}$ are just like the ones observed in Fig. la, b, g and h, that is, again a random height pattern is present in $W$ and the wavy height pattern is present in $W X$. As a result of this, the RMS roughness of the samples in this second situation is virtually identical to that of epiready wafers. 
Therefore, a roughening at the atomic scale of the germanium surface as a result of a high-temperature bake under $\mathrm{H}_{2}$ cannot be inferred from these results, as other authors have reported Additionally, as discussed for epiready samples, despite the differences found in morphology between these samples, both substrates meet the RMS criteria for excellent epitaxial growth and thus they do not aid in the identification of a clear cause for the degradation of morphology in the epilayers grown on $W X$ wafers.

\subsection{Morphology of hydride-exposed wafers}

Fig. le and $\mathrm{k}$ present topography scans of the $W$ and $W X$ wafers after the two bakes and the $\mathrm{PH}_{3}$ annealing at $640{ }^{\circ} \mathrm{C}$. The corresponding amplitude scans are shown in Fig. If and 1. The goal of this step in a typical heteronucleation procedure is to create a reconstructed surface consisting of group- $\mathrm{V}$ dimers, homogeneously oriented throughout the sample surface (i.e. single domain) covering the surface of the sample completely. For vicinal (100) substrates prepared in a MOVPE environment, the typical surface configurations of P-passivated Ge (or As-passivated $\mathrm{Ge})$ are either a $(2 \times 1)$ reconstruction $-\mathrm{P}$ dimers with bond axes parallel to step edges and dimer rows running perpendicular to step edges - or a ( $1 \times 2)$ reconstruction $-\mathrm{P}$ dimers with bond axes perpendicular to step edges and dimer rows running parallel to step edges [9]. The resulting phosphorus (or arsenic)-passivated surface is highly inert and self-limited to a single monolayer. Additionally, the hydride annealing helps to further decrease some carbon contamination that might have survived the hightemperature bake. Several works are available in the literature detailing the features of group-V passivated Ge surfaces, resulting from hydride annealings in a MOVPE growth environment, both for the case of $\mathrm{AsH}_{3}$ and also of $\mathrm{PH}_{3}$ surfaces . On the one hand, $\mathrm{AsH}_{3}$ has been reported to etch germanium significantly , even for temperatures of $540{ }^{\circ} \mathrm{C}$ far below the typical ranges used in heteroepitaxy $\left(600-650^{\circ} \mathrm{C}\right)$. Obviously, etching modifies the pre-existing substrate step structure, altering the desired morphology pattern prior to nucleation, being a source for crystallographic defects (APB) and inhomogeneities during epilayer growth. On the other hand, for $\mathrm{PH}_{3}$-exposed samples relevant etching has not been observed nor has a tendency toward step-bunching or faceting for temperatures up to $580{ }^{\circ} \mathrm{C} \quad$ Accordingly, Fig. le and k should again look very similar to Fig. lc and $\mathrm{i}$ (and thus to la and g) since they are expected to be the result of a set of steps which do not modify the morphology plus an inert exposure to $\mathrm{PH}_{3}$. However, Fig. le and $\mathrm{k}$ do not show the expected flat, smooth surface predicted. On the other hand, both samples present a random distribution of peaks of considerable height, as demonstrated by the high values of the surface kurtosis and the surface skewness in both cases (Table 1). Additionally, in this case, the differences are more marked between both samples, with the values of all the relevant roughness parameters being higher for the sample producing bad morphology, WX. For instance, surface skewness is positive and far from zero in both cases but its value on sample $W X$ roughly doubles that of sample $W$, suggesting an asymmetric height distribution (characteristic of a flat plane with sharp spikes) more pronounced for the $W X$ sample. A similar behaviour occurs for surface kurtosis, this time with a threefold difference in the magnitude of this parameter between both samples (related to this, notice the extreme peak roughly at the centre of Fig. Ik or the big bright spot roughly at the centre of the corresponding amplitude image 1.1). We believe that the differences encountered might be sufficient to justify the differences in the quality of the epilayers when an epitaxial routine is continued on these substrates. The presence of a larger amount of higher peaks in wafers $W X$ may spawn the formation of defects in subsequent epitaxial growth.

However, the reason why this degradation in morphology occurs is not fully understood yet. The presence of this peaky or mountain-valley morphology cannot be interpreted in terms of etching the germanium surface, since $\mathrm{PH}_{3}$ etch on $\mathrm{Ge}$ is negligible

Similar morphologies have been interpreted in terms of the interaction of carbon contamination with step-movement on $\mathrm{Ge}$ wafers $(\mathrm{C}$ is the only significant contaminant recurrently found in $\mathrm{Ge}$ surfaces baked at temperatures above $550{ }^{\circ} \mathrm{C}$ ) .

was proposed that carbon pins step movement and thus material tends to accumulate around points with a higher concentration of impurities thus creating the mountain-valley pattern. Additionally, it was quantified that carbon coverage on the surface is hard to remove below $8 \%$ by heating only (even at $700{ }^{\circ} \mathrm{C}$ ) and that it should not exceed $5 \%$ to guarantee good results for the heteroepitaxial growth . However, though the morphology reported in this work is similar to ours, this explanation does not fit well with our experiments. Particularly, if carbon was present on the surface of the samples the degradation in morphology should have already occurred during the high-temperature bake prior to hydride exposure, a fact that we did not observe.

Another possibility is that the morphology in Fig. le and $\mathrm{k}$ could be caused by a layer covering the germanium surface irregularly (i.e. carbon and/or oxygen compounds formed during air-exposure). When exposed to cracked phosphine, atomic hydrogen etches away this layer (which should be unaffected by high-temperature bakes to explain Fig. lc, d, i and j) producing a succession of peaks and valleys. It is unlikely that such a layer would be caused by the epiready process, so it is probably the result of air-exposure of the sample during the cutting process. Hence, to confirm this hypothesis a full wafer of WX-type was unpacked from its sealed box inside the reactor glovebox (i.e. in an $\mathrm{N}_{2}$ environment, with oxygen, water and hydrocarbon contents of below $1 \mathrm{ppm}$ ); it was immediately loaded into the reactor and our typical heteronucleation routine was carried out on it (this routine basically consists of the succession of steps analysed in this work plus the growth of 1 am of GalnP capped with 0.5 am of GaAs). The morphology results were surprisingly good, much better than when the growth takes place on pieces, though not totally free of crystallographic defects. However, the surface density of these defects was reduced considerably. In our opinion, this indicates two facts: (1) the exposure to the lab environment dramatically contributes to the degrading of the morphology (presumably by exposing the sample to carbon and/or oxygen contamination); and (2) the as-received epiready wafers have the seed for generating morphology problems since totally avoiding airexposure does not reduce crystallographic defects to zero.

\section{Summary and conclusions}

On many occasions, when growing $\mathrm{II}-\mathrm{V}$ semiconductors on germanium, unexpected differences between nominally identical substrates are encountered. We have identified a set of germanium substrates sharing the same specifications, coming from the same vendor but producing different results in terms of the morphology of the epilayers produced by the same epitaxial routine. We tried to gain some insight into this atypical behaviour by tracking the evolution in morphology of selected samples throughout the heteroepitaxial process. Particularly, using AFM, we analysed the morphology of (a) the epiready substrates; (b) the samples after high-temperature baking at $700{ }^{\circ} \mathrm{C}$; and (c) the samples after high-temperature baking at $700{ }^{\circ} \mathrm{C}$ plus a hydride 
$\left(\mathrm{PH}_{3}\right)$ annealing at $640{ }^{\circ} \mathrm{C}$. In the two first stages no big differences are found. Both substrates show excellent roughness parameters and seem to be unaffected by the temperature baking at $700{ }^{\circ} \mathrm{C}$. It is during the annealing in $\mathrm{PH}_{3}$ when the morphology degrades and the differences between both substrates become apparent. After phosphine exposure at $640{ }^{\circ} \mathrm{C}$, both substrates present a random distribution of peaks and valleys and a significant increase in the RMS roughness value. Despite this RMS roughness being similar in both samples $(0.6-0.7 \mathrm{~nm})$ several other roughness parameters show markedly higher values for the substrate producing bad morphology (namely, maximum peak-to-valley height, surface kurtosis and surface skewness), indicating that in this sample the peak density is higher as is their mean height. In other words, the hydride annealing causes a greater degradation of the surface of the substrates which will be prone to producing bad morphology in the heteroepitaxial process. On the other hand, neither the analysis of epiready wafers nor of high-temperaturebaked wafers have produced any useful hint in identifying troublesome substrates. The exact process behind the degradation in morphology is not yet fully understood. However, to assess the influence of air-exposure some experiments were repeated on full wafers not exposed to air. These experiments produced samples with a significantly lower density of defects indicating that (1) the exposure to the environment dramatically contributes to the degrading of morphology (presumably by exposing the sample to carbon and/or oxygen contamination); and (2) the as-received epiready wafers have the seed for generating morphology problems since totally avoiding air-exposure does not reduce crystallographic defects to zero.

\section{Acknowledgements}

AFM images included in this work were taken at the Centre for Electronic Microscopy "Luis Bru" (Universidad Complutense de Madrid) with the kind assistance of Mrs. Ana Soubrie.

Funding for this research was provided by the European Commission by Contract SES6-CT-2003-502620 (FULLPECTRUM project); by the Spanish Ministry of Education and Science (Ministerio de Education y Cientia) by projects GENESIS-FV (part of the CONSOLIDER-INGENIO 2010 programme), TEC2004-22300-E, TEC2005-02745 and TEC2007-29630-E; and by the Regional Government of Madrid (Comunidad de Madrid) by Contract S505/ENE/0310 (NUMANCIA programme).

\section{References}

B. Depuydt, A. Theuwis, I. Romandic, Mater. Sci. Semicond. Process. 9 (2006) 437.

K. Dessein, Role of the germanium substrate manufacturer in the CPV market, in: Presentation Given at 2007 Marburg Workshop on Concentrating Photovoltaic Optics and Power, downloadable at <http://concentrating-pv org/papers.html >.

Compound Semiconductor Industry September-December 2007 Review: Materials and Equipment, Strategy Analytics Report, October 2007.

K. Dessein, B. Depuydt, W. Geens and C. Quaeyhaegens, Zooming in on the surface of state-of-the-art germanium wafers, Paper Presented at ALTECH 07: Analytical Techniques for Semiconductor Materials and Process Characterization V, Munich (Germany), September 2007. Abstract downloadable at <http://www.chemie.uni-frankfurt.de/aac/ak kolbesen/english/conferences/ abstractfolder/Dessein_Umicore_abstr_ALTECH-2007_v2.pdf>.

S. Kalem, I. Romandic, A. Theuwis, Mater. Sci. Semicond. Process. 9 (2006) 753.

B. Galiana, I. Rey-Stolle, E. Barrigon, V. Corregidor, C. Algora, "Compositional analysis and evolution of defects formed on GalnP epilayers grown on Germanium," Proceedings of the ninth International Workshop on Beam Injection Assessment of Microstructures in Semiconductors (BIAMS08), Todelo (Spain), July 2008

D.J. Friedman, J.M. Olson, Prog. Photovoltaics Res. Appl. 9 (2001) 179

I. Horcas, R. Fernandez, J.M. Gomez-Rodriguez, J. Colchero, J. Gomez-Herrero, A.M. Baro, Rev. Sci. Instrum. 78 (2007) 013705.

J. Olson, W.E. McMahon, in: Proceedings of the Second World Conference on Photovoltaic Solar Energy Conversion, vol. 3,1998, p. 3540.

D.A. Allwood, R.T. Carline, N.J. Mason, C. Pickering, B.K. Tanner, P.J. Walker, Thin Solid Films 364 (2000) 33.

E. Marx, I.J. Malik, Y.E. Strasser, T. Bristow, N. Poduje, J.C. Stover, J. Vac. Sci. Technol.B 20(1) (2002) 31.

S. Sun, Y. Sun, Z. Liu, D.-Ick. Lee, S. Peterson, P. Pianetta, Appl. Phys. Lett. 88 (2006) 21903.

[13] S. Can, L. Li, T. Nguyen, H. Oj, R.F. Hicks, M. Yang, Surf. Sci. 395 (1) (1998) 69.

[14] Y Li, G. Salviati, M.M.G. Bongers, L. Lazzarini, L. Nasi, LJ. Giling, J. Crystal Growth 163 (1996) 195.

[15] S.M. Ting, J. Appl. Phys. 87 (5) (2000) 2618

] 16] S. Can, L Li, M.J. Begarney, D. Law, B.-K. Han, J. Appl. Phys. 85 (3) (1999) 2004.

[17] W.E. McMahon, J.M. Olson, in: Proceedings of the 12th American Conference on Crystal Growth Epitaxy, 2000.

[IS] W.E. McMahon, A.E. Kibbler, J.M. Olson, Surf. Sci. 571 (2004) 146.

[19] H. Okumura, T. Akane, S. Matsumoto, Appl. Surf. Sci. 125 (1998) 125. 